\title{
Solitary Plasmocytoma: 19-Year Retrospective Study and Review of the Literature
}

\author{
Marcio Tavares $^{1 *}$, Carolina Ferreira ${ }^{2}$, Renata Cabral ${ }^{3}$, Sergio Chacim ${ }^{1}$, Henrique Coelho ${ }^{4}$, Jorge \\ Coutinho $^{3}$, Jose Mario Mariz ${ }^{1}$, Angelo Oliveira ${ }^{2}$
}

${ }^{1}$ Department of Onco Hematology, Portuguese Institute of Oncology, Porto, Portugal

${ }^{2}$ Department of Radiotherapy, Portuguese Institute of Oncology, Porto, Portugal

${ }^{3}$ Department of Hematology, Porto General Hospital, Porto, Portugal

${ }^{4}$ Department of Hematology, Gaia-Espinho General Hospital, Gaia, Portugal

*Corresponding Author: Marcio Tavares, MD, Onco-Hematology Service, Portuguese Institute of Oncology in Porto, Rua António Bernardino Almeida, 4200-072 Porto, Portugal. E-mail: marcio.tavares@ipoporto.min-saude.pt

\begin{abstract}
Solitary Plasmacytoma (SP) is a rare plasma cell dyscrasia characterized by localized plasma cell infiltration. Radiotherapy is recommended as the treatment of choice. Epidemiological data is important to evaluate the effectiveness of treatment and the progression to Multiple Myeloma (MM). We described the clinical features and follow-up of 32 patients with SP treated with radiotherapy as primary treatment at our Department of Radiotherapy and we reviewed the literature on prognostic factors of progression to MM. Ninety percent of patients $(71 \%$ complete response and $19 \%$ partial response) responded to treatment. Fourteen patients progressed to MM with a median time of progression of 16, 9 months. With a median follow-up of 40 months, the 5 and 10-year estimated Overall Survey (OS) was 62, 6\% and 47, $7 \%$ respectively. Predictive factors of progression are still controversial. New factors are emerging in the fields of pathology, imagiology and immunology and thus we wait longer follow-up to confirm their predictive value. SP is highly radiosensitive and radiotherapy combines excellent control rates with minimal toxicity. However, it is a heterogeneous disease and approximately half of the patients will progress to MM. Identifying the patients more likely to progress would allow us to treat them in a different way.
\end{abstract}

Received Date: August 17, 2015

Accepted Date: October 27, 2015

Published Date: October 30, 2015

Citation: Tavares, M., et al. Solitary Plasmocytoma: 19-Year Retrospective Study and Review of the Literature. (2015) Int J Hematol Therap 1(2): 1-5.

DOI: $10.15436 / 2381-1404.15 .004$

Keywords: Multiple Myeloma; Plasma Cell Neoplasms; Plasmacytoma; Radiation Therapy

\section{Introduction}

Solitary plasmacytoma (SP) is characterized by localized monoclonal plasma cell infiltration without evidence of multiple myeloma and represents less than $10 \%$ of all plasma cell neoplasms ${ }^{[1-3]}$. These infiltrates arise either in the bone (Solitary plasmacytoma of the Bone- SBP) or in soft tissue (Extra medullary Plasmocytoma- EMP). Radiotherapy, alone or combined with surgery, is recommended as the treatment of choice for SP achieving excellent local control rates and high overall survival rates ${ }^{[4-8]}$. However, around $40 \%-50 \%$ of the patients will relapse and or progress to multiple myeloma (MM) with different rates for SBP and $\mathrm{EMP}^{[1,6,9,10]}$. The predictors of progression to $\mathrm{MM}$ are not consensual throughout the literature challenging the identification of those patients more likely to progress. Our Department of Radiotherapy has always played a central role in treating adults and children with cancer in Portugal. Except for single case reports, epidemiological data on SP have not been reported before in Portugal and it urges to characterize the patient population, to evaluate the effectiveness of radiation therapy and the risk of progression to MM and to improve the follow-up care of the patients. For this reason, the authors conducted a retrospective study to describe clinical 
features, the outcome and follow-up of a cohort of patients with $\mathrm{SP}$ treated at our institution. Additionally, we reviewed the literature and discussed known and recent risk factors of progression to MM.

\section{Material and Methods}

The Institutional Ethical Committee at the Portuguese Institute of Oncology - Porto reviewed and approved this study (reference number CES 209/2014). Thirty-two adult ( $>18$ years old) patients with De novo SP were treated consecutively at the Radiotherapy Department of our institution between January 1995 and December 2013. Patients treated for relapsed plasmocytoma were excluded. SP was defined based on the presence of the following criteria: 1) biopsy proven plasma cell tumor involving a single extramedullary or medullary site; 2) bone marrow aspirate or biopsy showing $<10 \%$ plasma cells; 3 ) no additional pathologic lesions in the skeletal diagnostic workup; 4) absence of MM-related anemia, hypercalcemia and renal disease; 5) low concentration of serum and urine monoclonal protein. Medical records were reviewed retrospectively for demographic details, tumor description, treatment plan, outcome and follow-up. Radiation doses of 15 to 46 Gy in 3-23 fractions were delivered with 2-5 Gy fractions. Radiotherapy-related toxicity greater than grade II of National Cancer Institute - Common Terminology Criteria Adverse Events v. 4.0 was considered significant. The response to treatment was defined as complete, partial, stable or progressive according to International Myeloma Working Group criteria $^{[11]}$. After completing their radiation therapy, patients were followed at three distinct Hematology Departments in the North of Portugal and adjuvant chemotherapy was decided according to each center policy. Assistant physicians at each center were asked to provide information on follow-up and treatment. The cohort was followed until June $30^{\text {th }}, 2014$. Data processing and statistical analysis were performed in Statistical Package for Social Sciences v.18. The Kaplan-Meier method was used to estimate overall survival and the differences between groups were assessed using the log-rank test. Values of $p<0,05$ were considered statistically significant.

\section{Results}

The median age at diagnosis of SP was 64,4 years $(30$ to 87 years) and the male-to-female ratio was $1: 1,29$. Twenty-eight patients $(87,5 \%)$ were diagnosed with SBP and vertebra was the most common location $(n=17)$. The remaining four patients $(12,5 \%)$ were diagnosed with EMP. Patients characteristics and anatomic location of SP are shown in table 1 and table 2, respectively. All patients received radiation therapy as front-line treatment. The median dose was $40 \mathrm{~Gy}$ (15 to $46 \mathrm{~Gy}$ ). Twelve patients were also treated with surgery for either diagnosis or symptomatic control - decompressive laminectomy and spine fusion. Data about drugs protocol was obtained in four of six patients that received adjuvant chemotherapy: Vincristine, Melphalan, Cyclophosphamide and Prednisone (VMCP), Melphalan and Prednisone (MP), Thalidomide and Dexametasone (TD) and Bortezomib and Dexametasone (VD). The main indication for adjuvant chemotherapy was SP size $\geq 50 \mathrm{~mm}$. The patient with EMP located at maxillary sinus developed mucositis grade III. Ninety percent of patients responded to treatment
(71\% complete response and $19 \%$ partial response). Nineteen patients relapsed: 14 patients $(43,5 \%)$ progressed to $\mathrm{MM}$ and the remaining five had relapse of SP. The median time to progression was 16, 9 months (9, 6 to 65,3 months). All patients that progressed to MM had been diagnosed with SBP and vertebral location was significantly associated with higher risk of progression comparing to non-vertebra location $(75 \%$ vs $16,7 \%$, $\mathrm{p}=0,02)$. After a median follow-up of 40 months $(0,5-189$, $1,12)$ patients $(34,4 \%)$ had died. Main causes of death were infectious disease $(n=3)$ and disease progression $(n=5)$. Among the patients treated with adjuvant chemotherapy, one progressed to $\mathrm{MM}$ and two were alive at the end of the study. Overall, the 5 and 10-year estimated overall survival was $62,6 \%$ and 47 , $7 \%$ respectively (figure 1). Patients that progressed to MM had shorter 10- year estimated OS comparing to patients that did not progressed (70, $8 \%$ vs 25, 5\%), although this difference was not statistically significant ( $\mathrm{p}=0,096$ ) (figure 2 ).

Table 1: Characteristics of 32 patients with solitary plasmacytoma

\begin{tabular}{|c|c|c|}
\hline Age & median & $64,4(30-87)$ \\
\hline \multicolumn{3}{|l|}{ Sex } \\
\hline Male (\%) & 18 & 56,3 \\
\hline \multicolumn{3}{|l|}{ Tumor size (\%) } \\
\hline$<50 \mathrm{~mm}$ & 25 & 78,9 \\
\hline$\geq 50 \mathrm{~mm}$ & 7 & 21,9 \\
\hline Microscopic & 22 & 68,7 \\
\hline \multicolumn{3}{|l|}{ LDH (\%) } \\
\hline Normal & 18 & 75 \\
\hline High & 6 & 25 \\
\hline NA & 8 & \\
\hline \multicolumn{3}{|c|}{ B2microglobulin(\%) } \\
\hline Normal & 16 & 80 \\
\hline High & 4 & 20 \\
\hline NA & 12 & \\
\hline \multicolumn{3}{|l|}{ Albumin (\%) } \\
\hline Normal & 18 & 78,3 \\
\hline Low & 5 & 21,7 \\
\hline NA & 9 & \\
\hline \multicolumn{3}{|l|}{ Treatment (\%) } \\
\hline RT only & 14 & 43,8 \\
\hline RT + Surgery & 12 & 37,5 \\
\hline $\mathrm{RT}+\mathrm{CT}$ & 6 & 18,8 \\
\hline \multicolumn{3}{|l|}{ RT dose $(\%)$} \\
\hline$<40 \mathrm{~Gy}$ & 10 & 31,3 \\
\hline$\geq 40 \mathrm{~Gy}$ & 22 & 68,8 \\
\hline
\end{tabular}

Abbreviations: CT - chemotherapy; LDH - lactate dehydrogenase; NA - not available; RT - radiation therapy. 
Table 2: Anatomic location of extramedullary and bone plasmacyytomas

\begin{tabular}{|c|c|c|c|}
\hline SBP & 28 & EMP & 4 \\
\hline Vertebra & 17 & Nasopharynx & 3 \\
\hline Iliac & 4 & Paranasal sinus & 1 \\
\hline Upper limb & 2 & & \\
\hline Lower limb & 2 & & \\
\hline Thorax gride + sternum & 3 & & \\
\hline Skull & 1 & & \\
\hline
\end{tabular}

Abbreviations: EMP - extramedullary plasmacytoma; SBP - solitary bone plasmacytoma.

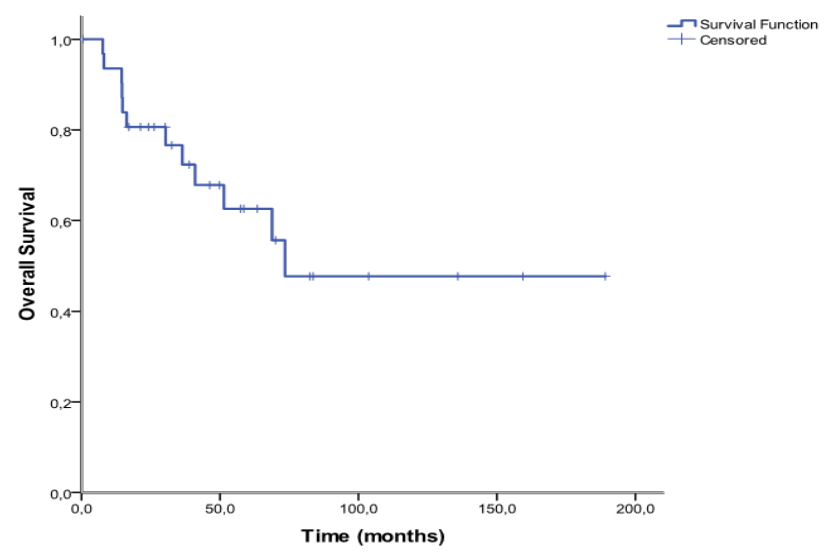

Figure 1: Overall survival in 32 patients with solitary plasmacytoma.

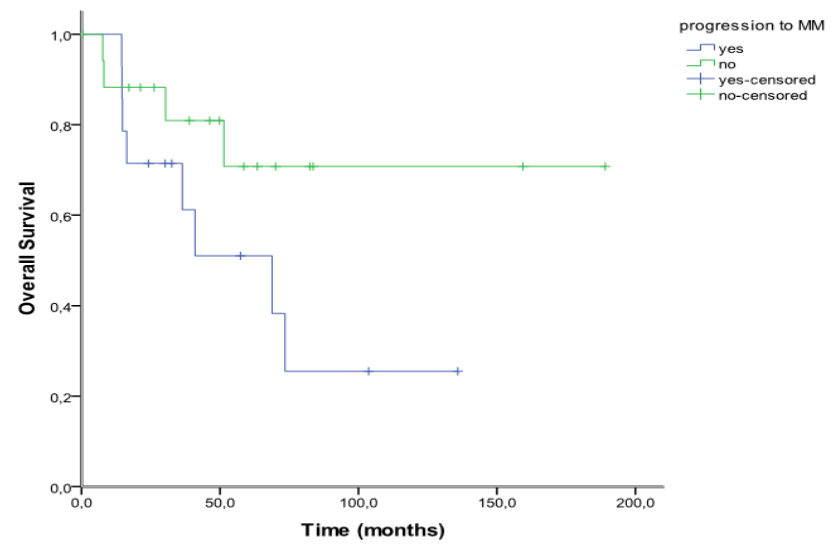

Figure 2: Overall survival in 32 patients with solitary plasmacytoma that progressed vs not progressed to multiple myeloma.

\section{Discussion}

SP are rare tumors of plasma cell origin classified as SBP or EMP according to its location. According to a United States population-based study, SP is 16 times less frequent than $\mathrm{MM}^{[3]}$. A full work-up at diagnosis is mandatory to exclude systemic multiple myeloma and the following investigations should be performed in all patients: complete blood count, biochemical screen, serum and urine electrophoresis and immunofixation, bone marrow aspirate and trephine biopsy and complete skeletal survey ${ }^{[12,13]}$. Although being a typical disease of the elderly, there are some case reports regarding pediatric patients ${ }^{[14-16]}$. In this study, we present the largest Portuguese cohort of consecutive patients with SP treated with radiation therapy and followed for a median time of 40 months $((0,5-189,1), 12$ patients $(34,4 \%)$ had died., 1 months). SBP was diagnosed in $87,5 \%$ of the patients and the majority of these tumors arose in axial skeleton especially the vertebra. EMP was less common (12, 5\%) and arose predominantly in the head and neck.

Radiotherapy is recommended as the treatment of choice for SP but the optimal radiation dose has not been yet established. Even though a dose of 40-50 Gy in 4-5 weeks is generally recommended, in clinical practice, some patients don't tolerate such high doses ${ }^{[1,12]}$. Furthermore, some studies reported no dose-relationship in radiation therapy for plasmacytoma $a^{[1,6,17]}$. In our study, $31,3 \%$ of the patients received less than the recommended dose. However, the median radiation dose was $40 \mathrm{~Gy}$ and lower dose did not associated with higher risk of progression $(p=0,096)$. Ninety percent of the patients responded to treatment confirming that SP is a highly radiosensitive disease and that excellent control rates can be achieved with radiation therapy. Some patients need surgery for pain control, decompressive laminectomy, spine fusion or fixation of a long bone ${ }^{[7,12]}$. Adjuvant chemotherapy may be considered in other patients but it does not inhibit progression of SP to MM nor offer any survival benefit ${ }^{[6,18]}$. The role of novel agents (bortezomib, thalidomide and lenalidomide) was recently evaluated by the Greek myeloma study group. This study showed that patients treated with chemotherapy or novel agents-based regimens lived shorter and had more toxicity than patients that received radiotherapy only. The authors consider both chemotherapy and novel-agents based therapy as "overtreatment"[4]. Overall, our patients with SP presented prolonged OS. Patients that progressed to MM showed a tendency for shorter 10-year estimated OS comparing to patients that did not progressed. We could not show any significant difference between the two groups partially because of limited sample size and or short follow-up.

More than $40 \%$ of patients progress to MM with a median time of 2 to 4 years. It is important to identify high risk patients because progression to $\mathrm{MM}$ is the most important predictive factor for overall survival in patients with SP. However, factors that influence the risk and frequency of progression to MM (age, tumor size, radiation dose, M-protein levels, post-radiotherapy persistent of M-protein) are reported inconsistently in the literature (table 3). Unfortunately, the small size of our population precluded the analysis of such risk factors. Many studies have reported older age as an independent prognostic factor of progression to $\mathrm{MM}^{[5,10]}$. However, other studies have not confirmed such relation and, in MD Anderson Cancer Institute, younger patients showed a higher risk of progression ${ }^{[9]}$. Positive serum M-protein by immunofixation is observed in 30 to $70 \%$ of patients with SP. It is still unclear whether secretory or unsecretory SP associated with improved outcomes ${ }^{[9,19]}$. Many patients maintain abnormal levels of M-protein after radiation therapy and some authors regard post-treatment persistence of myeloma protein as an adverse prognostic factor ${ }^{[7,20]}$. However, that was based mainly in single-center studies and a national multicenter study did not confirm its predictive value ${ }^{[4]}$. The literature also contains conflicts on the predictive impact of lesion size on the progression to $\mathrm{MM}^{[5,21]}$. 
Solitary Plasmocytoma- Study \& Review

Table 3: Results of the largest series of solitary plasmacytoma reported in the literature

\begin{tabular}{|c|c|c|c|c|c|}
\hline Author & $\begin{array}{l}\text { No. Patients } \\
(\text { SBP + EMP) }\end{array}$ & $\begin{array}{l}\text { RT dose, Gy (min - } \\
\text { max) }\end{array}$ & $\begin{array}{l}\text { Progression to } \mathrm{MM} \\
(\%)\end{array}$ & Negative predictors of progression* & $\begin{array}{l}\text { Follow-up, } \\
\text { median }\end{array}$ \\
\hline Holland et al, $1992^{6}$ & $46(32+14)$ & $46,10(16,11-62,00)$ & $22(47,8)$ & None & 66 months \\
\hline Tsang et al, $2001^{21}$ & $46(32+14)$ & 35 & $22(47,8)$ & Older age; bone SP location & 7,9 years \\
\hline Wilder et al, $2002^{20}$ & $60(60+0)$ & $46(30-70)$ & MFS $38 \%$ at 10 years & Post-RT persistence of M-protein & 7,8 years \\
\hline Ozsahin et al, $2006^{1}$ & $258(206+52)$ & $40(20-66)$ & $117(45,3 \%)$ & Bone SP location & 56 months \\
\hline Knobel et al, $2006^{10}$ & $206(206+0)$ & $40(20-64)$ & $104(50,5)$ & Older age & 54 months \\
\hline Kilciksiz et al, $2008^{5}$ & $80(57+23)$ & $46(32,5-64)$ & $25(31,2)$ & Old age & 2,41 years \\
\hline Reed et al, $2011^{9}$ & $84(59+25)$ & $45(36-53,4)$ & $38(54)$ & $\begin{array}{l}\text { Young age; bone SP location; positive } \\
\text { M-protein at diagnosis. }\end{array}$ & 64 months \\
\hline Guo et al, $2013^{19}$ & $66(45+21)$ & 50 & $18(27,3)$ & Positive M-protein at diagnosis & 48 months \\
\hline $\begin{array}{l}\text { Katodritou et al, } \\
2014^{4}\end{array}$ & $97(65+23)$ & $40(24-55)$ & $24(24,7)$ & Immunoparesis at initial diagnosis & 5 years \\
\hline Present study & $32(28+4)$ & $40(15-46)$ & $14(43,5)$ & None & 40 months \\
\hline
\end{tabular}

Abbreviations: Max - maximum; Min - minimum; MM - multiple myeloma; M-protein - monoclonal protein; SP - solitary plasmacytoma; RTradiation therapy. *Based on multivariate analyses.

New predictive factors have been proposed and new investigations have been suggested. Kumar et al showed that highgrade angiogenesis associated with higher risk of progression to $\mathrm{MM}$ and shorter progression-free survival ${ }^{[22]}$. Immunoparesis has been reported in 25 to $87 \%$ of patients diagnosed with $\mathrm{SP}^{[4]}$. Even though its pathogenesis in MM remains unclear, it was the most powerful predictor of progression to $\mathrm{MM}$ in a cohort of 97 patients followed for a median of five years ${ }^{[4]}$. Patients with an abnormal serum free light chain ratio at diagnosis showed a higher risk of progression to $\mathrm{MM}$ and a possible prognostic role of monoclonal light chains was proposed. A model risk incorporating an abnormal serum free light chain ratio and the persistence of detectable M-protein was created and patients having both risk factors showed a risk of progression at 5 years of $62 \%$ compared to $13 \%$ of patients with no risk factors ${ }^{[23]}$. Novel imaging techniques such as Magnetic Resonance Imaging (MRI) and Positron Emission Tomography With Computed Tomography (PET-TC) have been suggested in the work-up of MM and related diseases ${ }^{[24,25]}$. More recently two independent groups showed that Multiparameter Flow Cytometry (MFC) could define high-risk patients. Although using different methodology in flow citometry, the results were similar: approximately $70 \%$ of the patients with SBP and positive flow citometry progressed to MM with a median time of 26 months comparing to $6-12,5 \%$ of the patients without occult bone marrow disease $\mathrm{e}^{[2,27]}$. Despite being potentially valuable in the management of SBP patients, MFC may be less informative in EMP.

\section{Conclusion}

As a conclusion, $\mathrm{SP}$ is a heterogeneous disease and radiation therapy combines excellent local control rates with minimal toxicity. However, progression to MM remains an important problem and classical risk factors have not helped us tackle it. New factors are emerging and we await larger series with longer follow-up to confirm their predictive value. Until then, all patients with SP should be followed-up closely and treated equally.
Acknowledgment: This research was supported by the Liga Portuguesa Contra o Cancro - Núcleo Regional Norte.

\section{References}

1. Ozsahin, M., Tsang, R.W., Poortmans, P., et al. Outcomes and patterns of failure in solitary plasmacytoma: A multicenter Rare Cancer Network study of 258 patients. (2006) Int J Radiat Oncol Biol Phys 64(1): 210-217.

2. Dimopoulos, M.A., Hamilos, G. Solitary bone plasmacytoma and extramedullary plasmacytoma. (2002) Curr Treat Options Oncol 3(3): 255-259.

3. Dores, G.M., Landgren, O., Mcglynn, K.A., et al. Plasmacytoma of bone, extramedullary plasmacytoma, and multiple myeloma: Incidence and survival in the United States. (2009) Br J Haematol 144(1): 86-94.

4. Katodritou, E., Terpos, E., Symeonidis, A.S., et al. Clinical features, outcome, and prognostic factors for survival and evolution to multiple myeloma of solitary plasmacytomas: A report of the Greek myeloma study group in 97 patients. (2014) Am J Hematol 89(8): 803-808.

5. Kilciksiz, S., Celik, O.K., Pak, Y., et al. Clinical and prognostic features of plasmacytomas: A multicenter study of Turkish Oncology Group-Sarcoma Working Party. (2008) Am J Hematol 83(9): 702-707.

6. Holland, J., Trenkner, D.A., Wasserman T.H., et al. Plasmacytoma: Treatment results and conversion to myeloma. (1992) Cancer 69(6): 1513-1517.

7. Kumar, S. Solitary plasmacytoma: Is radiation therapy sufficient? (2008) Am J Hematol 83(9): 695-696.

8. Hughes, M., Soutar, R., Lucraft, H., et al. Guidelines on the diagnosis and management of solitary plasmacytoma of bone, extramedullary plasmacytoma and multiple solitary plasmacytomas: 2009 update. (2009) British Committee for Standards in Haematology, London

9. Reed, V., Shah, J., Medeiros, L.J., et al. Solitary plasmacytomas: Outcome and prognostic factors after definitive radiation therapy. (2011) Cancer 117(19): 4468-4474.

10. Knobel, D., Zhouhair, A., Tsang, R.W., et al. Prognostic fac- 
tors in solitary plasmacytoma of the bone: A multicenter Rare Cancer Network study. (2006) BMC Cancer 6: 118.

11. Durie, B.G.M., Harousseau, J.L., Miguel, J.S., et al. International uniform response criteria for multiple myeloma. (2006) Leukemia 20(9): 1467-1473.

12. Kilciksiz, S., Karakoyun-Celik, O., Agaoglu, F.Y., et al. A review for solitary plasmacytoma of bone and extramedullary plasmacytoma. (2012) Sci World J 2012: 895765.

13. Moreau, P., San, M.J., Ludwig, H., et al. Multiple myeloma: ESMO clinical practice guidelines for diagnosis, treatment and follow-up. (2013) Ann Oncol (SUPPL 6): vi133-137.

14. Dumesnil, C., Schneider, P., Dolgopolov, I., et al. Solitary bone plasmocytoma of the spine in an adolescent. (2006) Pediatr Blood Cancer 47(3): 335-338.

15. Panteli, K., Tsiara, S., Bourantas, K.L. Solitary bone plasmacytoma in a young patient. (2002) J Exp Clin Cancer Res 21(1): 139-141.

16. Mann, G., Trebo, M.M., Minkov, M., et al. Extramedullary plasmacytoma of the adenoids. (2007) Pediatr Blood Cancer 48(3): 361-362.

17. Frassica, D.A., Frassica, F.J., Schray, M.F., et al. Solitary plasmacytoma of bone: mayo clinic experience. (1989) Int J Radiat Oncol Biol Phys 16(1): 43-48.

18. Galieni, P., Cavo, M., Avvisati, G., et al. Solitary plasmacytoma of bone and extramedullary plasmacytoma: Two different entities? (1995) Ann Oncol 6(7): 687-691.

19. Guo, S.Q., Zhang, L., Wang, Y.F., et al. Prognostic factors associated with solitary plasmacytoma. (2013) Onco Target Ther 6: 1659-1666
20. Wilder, R.B., Ha, C.S., Cox, J.D., et al. Persistence of myeloma protein for more than one year after radiotherapy is an adverse prognostic factor in solitary plasmacytoma of bone. (2002) Cancer 94(5): 1532-1537.

21. Koukourakis, M.I., Giatromanolaki, A., Kakolyris, S., et al. Solitary plasmacytoma treated with radiotherapy: Impact of tumor size on outcome. (2001) Int J Radiat Oncol Biol Phys 50(1): 113-120.

22. Kumar, S., Fonseca, R., Dispenzieri, A., et al. Prognostic value of angiogenesis in solitary bone plasmacytoma. (2003) Blood 101(5): 1715-1717.

23. Dingli, D., Kyle, R.A., Rajkumar, S.V., et al. Immunoglobulin free light chains and solitary plasmacytoma of bone. (2006) Blood 108(6): 1979-1983.

24. Fouquet, G., Guidez, S., Herbaux, C., et al. Impact of initial FDG-PET/CT and serum-free light chain on transformation of conventionally defined solitary plasmacytoma to multiple myeloma. (2014) Clin Cancer Res 20(12): 3254-3260.

25. Warsame, R., Gertz, M.A., Lacy, M.Q., et al. Trends and outcomes of modern staging of solitary plasmacytoma of bone. (2012) Am J Hematol 87(7): 647-651.

26. Paiva, B., Chandia, M., Vidriales, M.B., et al. Multiparameter flow cytometry for staging of solitary bone plasmacytoma: New criteria for risk of progression to myeloma. (2014) Blood 124(8): 1300-1303.

27. Hill, Q.A., Rawstron, A.C., De Tute, R.M., et al. Outcome prediction in plasmacytoma of bone: A risk model utilizing bone marrow flow cytometry and light-chain analysis. (2014) Blood 124(8): 1296-1299. 